\title{
Lattice Effects in the Complex Subband Dispersion of 2DEG Semiconductor Waveguide Structures Subjected to a Perpendicular Magnetic Field*
}

\begin{abstract}
G. EDWARDS and D. K. FERRY
Center for Solid State Electronics Research, Arizona State University, Tempe, Arizona 85287-6206, U.S.A.

In modeling waveguide magneto-transport experiments (in a quasi-two dimensional electron gas), it is important to have knowledge of the electronic states in a magnetic field perpendicular to the plane of the waveguide confinement potential. We present numerical results, within a lattice model, for the full complex subband dispersion of a rectangular waveguide. The form of our numerical real-subband solutions agrees well with analytical real solutions. However, some of our numerical evanescent solutions have a different topology from the analytic evanescent solutions near the bandedges. We argue that our evanescent solutions, although consistent with the symmetry of the lattice model and mode conservation in the restricted Hilbert space of the discretized Hamiltonian, yield different results that are forbidden in the continuum solutions. This is a concern for numerical solutions.
\end{abstract}

Keywords: lattice model, waveguide, complex dispersion, subbands

In trying to understand mesoscopic waveguide magneto-transport experimental results, it is necessary to have knowledge both of the electronic states in the presence of a magnetic field, perpendicular to the plane of the 2DEG, and of the waveguide confinement potential [1]. The Schrödinger equation (SE), including the $\mathbf{B}$ field and the confinement potential, can be solved by a discretization procedure and subsequently working with a lattice model [2-4]. We examine the full magneto-electric complex subband dispersion of a rectangular waveguide, including both the real bands and the complex evanescent bands. Usually, when considering the states of a rectangular waveguide, only the real dispersion is calculated [1]. However, the full complex dispersion is needed in treating a heterogeneous structure, such as a ballistic cavity or disordered quantum wire in a $\mathbf{B}$ field [1-4].
We consider a 2D symmetric rectangular waveguide geometry with hard-wall confinement at $y= \pm W / 2$ and free motion along the $x$ axis. The 2D $\mathrm{SE}$, including the presence of a perpendicular $\mathbf{B}$ field in the Landau gauge $\mathbf{A}(y)=(-B y, 0)$, is discretized with lattice constant $a$. The discretized SE then consists of a set of difference equations, and is equivalent to a 2D tight-binding (TB) model in which the hopping matrix element along $x$ is modified by a Peierls' phase factor due to the presence of the $\mathbf{B}$ field [2]. Considering $N_{y}$ lateral sites inside the waveguide, where $N_{y}$ is given by $W=\left(N_{y}+1\right) a$, and therefore 'truncating' the TB equations, effectively imposes hard-wall waveguide boundary condition at $y= \pm W / 2$ [2].

The equation of motion along $x$, which relates the wavefunction amplitudes on longitudinally neighboring columns of lateral sites, can be written as a

* Work supported by NEDO under an International Joint Research Program. 
$2 N_{y} \times 2 N_{y}$ transfer matrix (T-matrix) [2]. Crystal translational symmetry is maintained along $x$ as the hard walls are along $y$ and the Landau gauge is chosen to be $y$ dependent so the solution along $x$ is Blochlike, i.e. $\exp \left(i k_{x} l a\right)$ at $x=l a$ [2]. Inserting this Bloch form of solution along $x$ into the set of T-matrix equations results in a single $2 N_{y} \times 2 N_{y}$ non-Hermitian Tmatrix eigenvalue equation with the eigenvalues expressed as $\exp \left(i k_{x} a\right)$, for a given energy $E$ [2]. There are thus $2 N_{y} k$ wavevector solutions for each $E$, which by symmetry can be divided into $N_{y}$ forward and $N_{y}$ backward waves as discussed below [5]. From general tight-binding theory, $N_{y}$ lateral sites (when lateral hard wall boundary conditions are applied) give rise to $N_{y}$ bands [5]. Considering charge conjugation symmetry and reflection symmetry about the $x$ axis of the waveguide within the lattive model, the wavevector solutions for energy $E$ occur in quartets, i.e. $\left\{k,-k, k^{*},-k^{*}\right\}$ [5]. A general complex $k$ is represented as $k=\alpha+i \beta$, where $\alpha$ and $\beta$ are purely real (Re). For $k$ purely $\mathrm{Re}$, i.e. propagating states, there are only 2 distinct solutions $k= \pm \alpha$ which can be built from this quartet of solutions. These purely real states can be classified into forward $(f) k=+\alpha$ and backward $(b) k=-\alpha$ states propagating along $+x$ and $-x$ respectively. Similarly for $k$ purely imaginary (Im), i.e. evanescent states, there are also only 2 distinct solutions $k= \pm i \beta$. These states can also be classified into $f(k=+i \beta)$ and $b(k=-i \beta)$ states, decaying along $+x$ and $-x$ respectively. For $k$ complex, which is also an evanescent wave, the quartet of possible solutions are now all distinct and may be grouped $(k=+\alpha \pm i \beta$, $k=-\alpha \pm i \beta$ ) into 2 bands each with a $f$ and $b$ state. The $f / b$ classification for complex states is done in the same fashion as for purely imaginary states. In the bandstructure graphs of this paper, we only plot the $N_{y} f$ states. The complex pair of states $k= \pm \alpha+i \beta$ are actually plotted as $k=| \pm \alpha|+i \beta=+\alpha+i \beta$, so appear as degenerate in the graphs of this paper.

In modeling magneto-transport, we need to be able to describe the states up to and at the Fermi level [1]. The de Broglie wavelength decreases with increasing energy. Thus, if we choose the lattice constant sufficiently small so that states at the Fermi energy are in the continuum limit, i.e. the Fermi wavelength satisfies $\lambda_{F}>>a$, then the lower energy states with fewer oscillations automatically should be well described by the lattice model. In a GaAs 2DEG structure at a typical doping density, the Fermi energy $E_{F} \sim 16-20 \mathrm{meV}$ and for $E_{F}=20 \mathrm{meV}$ then $\lambda_{F}=33.5 \mathrm{~nm}$ [1]. In our calculations we choose $a=5 \mathrm{~nm}\left(\lambda_{F}=6.7 a\right)$ and $a=2.5 \mathrm{~nm}\left(\lambda_{F}=13.4 a\right)$ and expect in both cases the discretization to be fine enough to be in the continuum limit.

First consider the purely real wavevector dispersion and the convergence properties of the solutions within the lattice model. Figure 1 shows the real wavevector dispersion for $W=100 \mathrm{~nm}$ and $B=1 \mathrm{~T}$, a

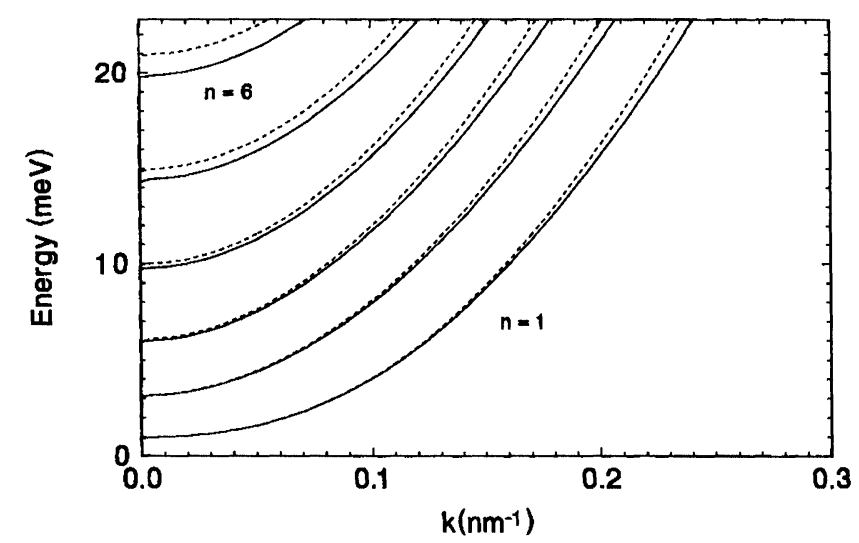

FIGURE 1 The real wavevector subband dispersion, along $x$, for a waveguide in a 'weak' applied $\mathbf{B}$ field with $W=100 \mathrm{~nm}, B=1 \mathrm{~T}$. The solid lines are for a lattice constant $a=5 \mathrm{~nm}$ and the dashed lines are for $a=2.5 \mathrm{~nm}$ 
'weak' field regime [1]. In Fig. 1, the solid lines are for $a=5 \mathrm{~nm}$ and the dashed lines are for $a=2.5 \mathrm{~nm}$. The 'weak' field subband dispersions have a parabolic dispersion, as expected from first-order perturbation theory, which gives a diamagnetic shift of the dispersion of the unperturbed system [6]. The topology of the real subband dispersions is seen not to depend on $a$. The $a=2.5 \mathrm{~nm}$ dispersions lie slightly above the $a=5 \mathrm{~nm}$ dispersion. The difference between the $a=5 \mathrm{~nm}$ and $a=2.5 \mathrm{~nm}$ dispersions becomes greater for the higher subbands, due to the 'rapidly' oscillating nature of the solution for the 'higher' states. Thus, as expected from the choice of $a$, which is taken from the outset to be fine enough to describe states up to $E_{F} \sim 20 \mathrm{meV}$, our numerical real subband dispersions are reasonably well converged. However, as we will see, this is not the situation for the evanescent states.

In Fig. 2, we display the full complex bandstructure [5] in the 'weak' field case for $W=100 \mathrm{~nm}, B=1 \mathrm{~T}$ and $a=5 \mathrm{~nm}$. There are a total of $N_{y}=19$ bands for this lattice constant. The 6 real parabolic wavevector dispersions are clearly visible. At energies just below the $n=1 \rightarrow n=6$ bandedges, the analytic continuation [5] is a purely imaginary band with a parabolic dispersion 'downwards'. First-order perturbation theory also yields this type of dispersion [6]. However, slightly below the $n=1 / n=2$ bandedges at $E \sim 0.75$ $\mathrm{meV}$, the $n=1 / n=2$ purely imaginary bands merge, forming a complex band [5]. For $E<0.75 \mathrm{meV}$, this band remains complex. This behavior is not concordant with the purely imaginary solution obtained from perturbation theory, although a complex band is still an evanescent wave in character. This merging phenomena does conserve the number of solutions. Before the merging, there are 2 distinct $n=1 / n=2$ purely $\operatorname{Im}$ bands, i.e. 4 solutions including $f$ and $b$ states $k=\left\{ \pm i \beta_{1}, \pm i \beta_{2}\right\}$. After the merging, there is a complex quartet of solutions, i.e. a pair of bands $k=$ $+\alpha \pm i \beta$ and $k=-\alpha \pm i \beta$. Thus, two distinct purely imaginary bands merge and become a complex pair of bands, which conserves the total number (4) of solutions.

At an energy of $E \sim-5 \mathrm{meV}$, purely imaginary bands $n=3 / n=4$ and $n=12 / n=13$ merge, forming 2 pairs of complex bands, with the appearance of 2 new real parts in the $\operatorname{Re}(k)$ dispersion for $\mathrm{E}<-5 \mathrm{meV}$. The 3 'vertical lines' in the $\operatorname{Re}(k)$ dispersion over the total energy range are the Re parts of 3 complex pairs of bands. The 3 outermost curves in the $\operatorname{Im}(k)$ dispersion

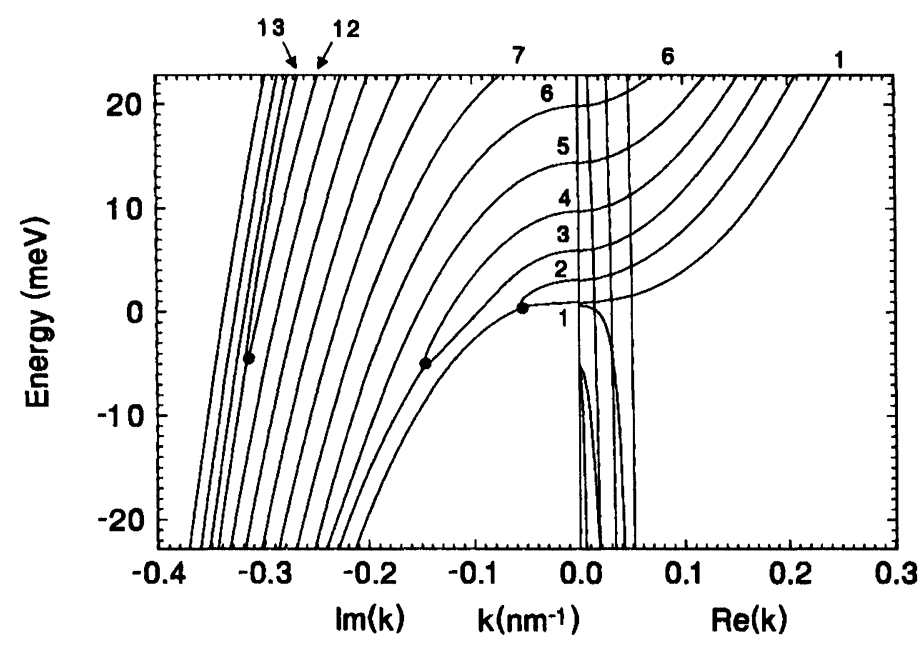

FIGURE 2 The full complex bandstructure dispersion, along $x$, for a waveguide subjected to a 'wea' $\mathbf{B}$ field with $W=100$ nm, $B=1 \mathrm{~T}$ and for $a=5 \mathrm{~nm}$ where there are a total of 19 bands. The imaginary dispersion has several band mergings, which are annotated with a black dot, where the purely imaginary solutions become complex 
are the Im parts of these 3 complex pairs of bands. At $E=23 \mathrm{meV}$, there are 6 purely Re bands, 7 purely $\mathrm{Im}$ bands and 3 pairs of complex bands giving a total of 19 bands, the number of lateral sites. For $E<23 \mathrm{meV}$ the full complex bandstructure evolves in a way compatible with the conservation of a total number of $N_{y}=19$ bands. For $a=2.5 \mathrm{~nm}$, the $n=3 / n=4$ band merging occurs deeper into the analytic continuation region and the $n=12 / n=13$ bands remain purely Im over the entire enegry range.

Our results show that, for a chosen sufficiently small to represent an oscillatory waveguide solution at the Fermi energy, the real subband dispersion is well described up to the Fermi energy. The real dispersion topology is also stable, on decreasing $a$. However, the evanescent bands are well described only for a range of energy, below their bandedges, considerably less than the Fermi energy. The topology of the evanescent bands is sensitive to decreasing $a$. The evanesent bandstructure deep into the analytic continuation region, although an inaccurate 'depiction' of the continuum bands, are valid lattice-model solutions. These 'spurious' evanescent solutions are allowed within the lattice model, from symmetry and solution number conservation considerations and their existence are equivalent to a basis size truncation effect [5]. To describe the waveguide dispersion up to an equal energy above and below a bandedge, the evanescent dispersion region will require a smaller $a$ than the real dispersion region for results of similar accuracy. This is because, at equal energies below and above a bandedge, the evanescent wave is a more rapidly varying function spatially than the propagating wave. For situations where evanescent waves can be strongly excited, such as encountered in the calculations of electronic states of heterogeneous waveguide structures [4], care must be taken so that the discretization really is fine enough to treat the evanescent dispersion correctly. There will be a trade off between evanescent state dispersion accuracy and intensive memory/CPU computational requirements.

\section{References}

[1] C. W.J. Beenakker and H. Van Houten, Solid State Phys. 44, 1, (1991)

[2] T. Ando, Phys. Rev. B 44, 8017, (1991)

[3] G. Edwards, A. Grincwajg and D. K. Ferry, Jpn. J. Appl. Phys. 34, (1995), Part 1, No. 8B(August)

[4] G. Edwards, A. Grincwajg and D. K. Ferry, Surf. Sci, in press

[5] D. L. Smith and C. Mailhiot, Rev. Mod. Phys. 62, 1, (1990)

[6] J. C. Maan in 'Physics and Applications of Quantum Wells and Superlattices', ed. E. E. Mendez and K. Von Klitzing, Plenum Press, 1987

\section{Biography}

Gerard Edwards received his B.A. degree in physics from Oriel College, Oxford, U.K. in 1988 and his $\mathrm{Ph} . \mathrm{D}$. in theoretical physics from the University of Exeter, U.K., in 1992. He subsequently pursued postdoctoral research work in the area of theoretical semiconductor physics at the University of Nottingham, U.K., and Arizona State University. Currently he is a development engineer at Silvaco International, engaged in the incorporation of quantum effects into commercial semiconductor device simulators. 

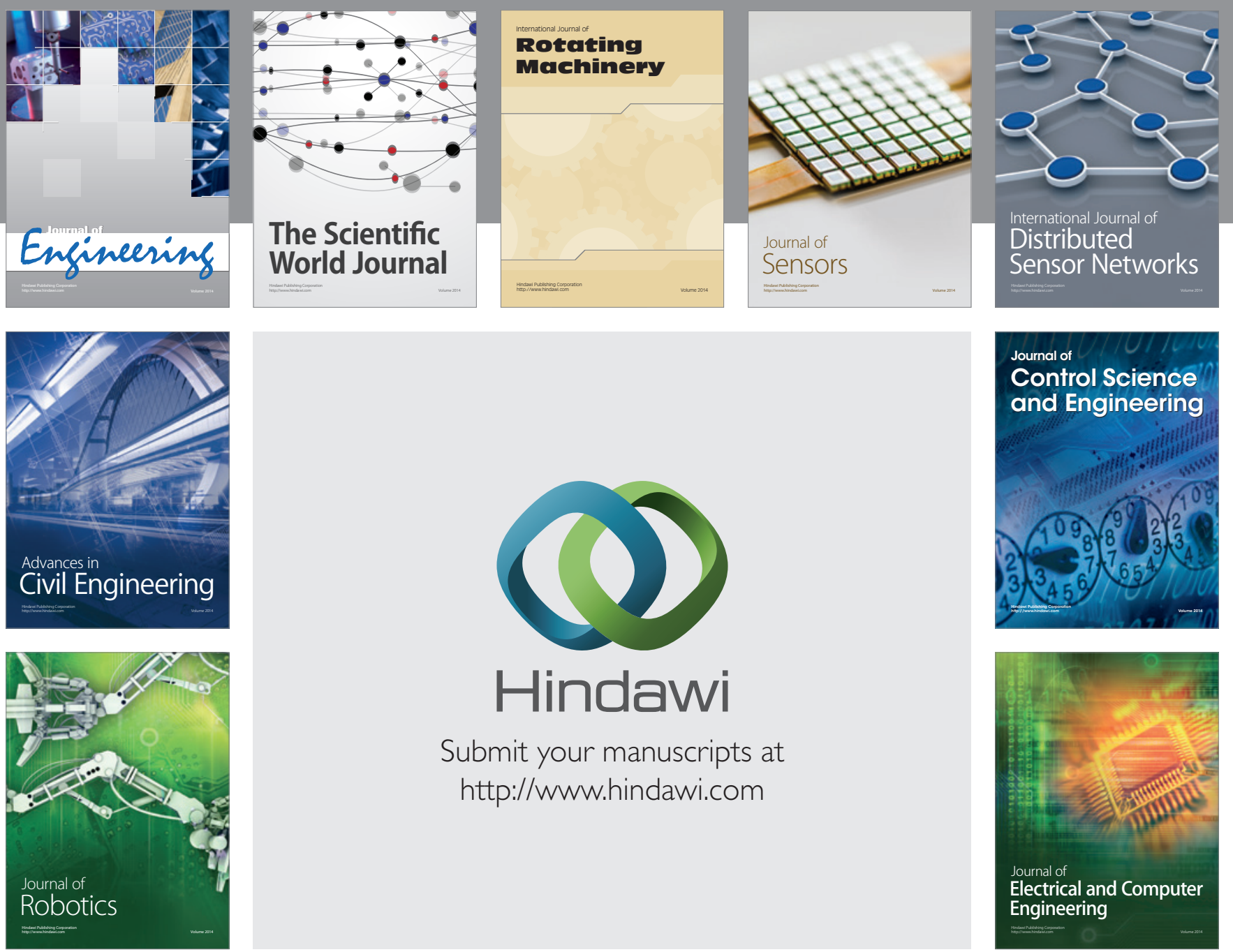

Submit your manuscripts at

http://www.hindawi.com
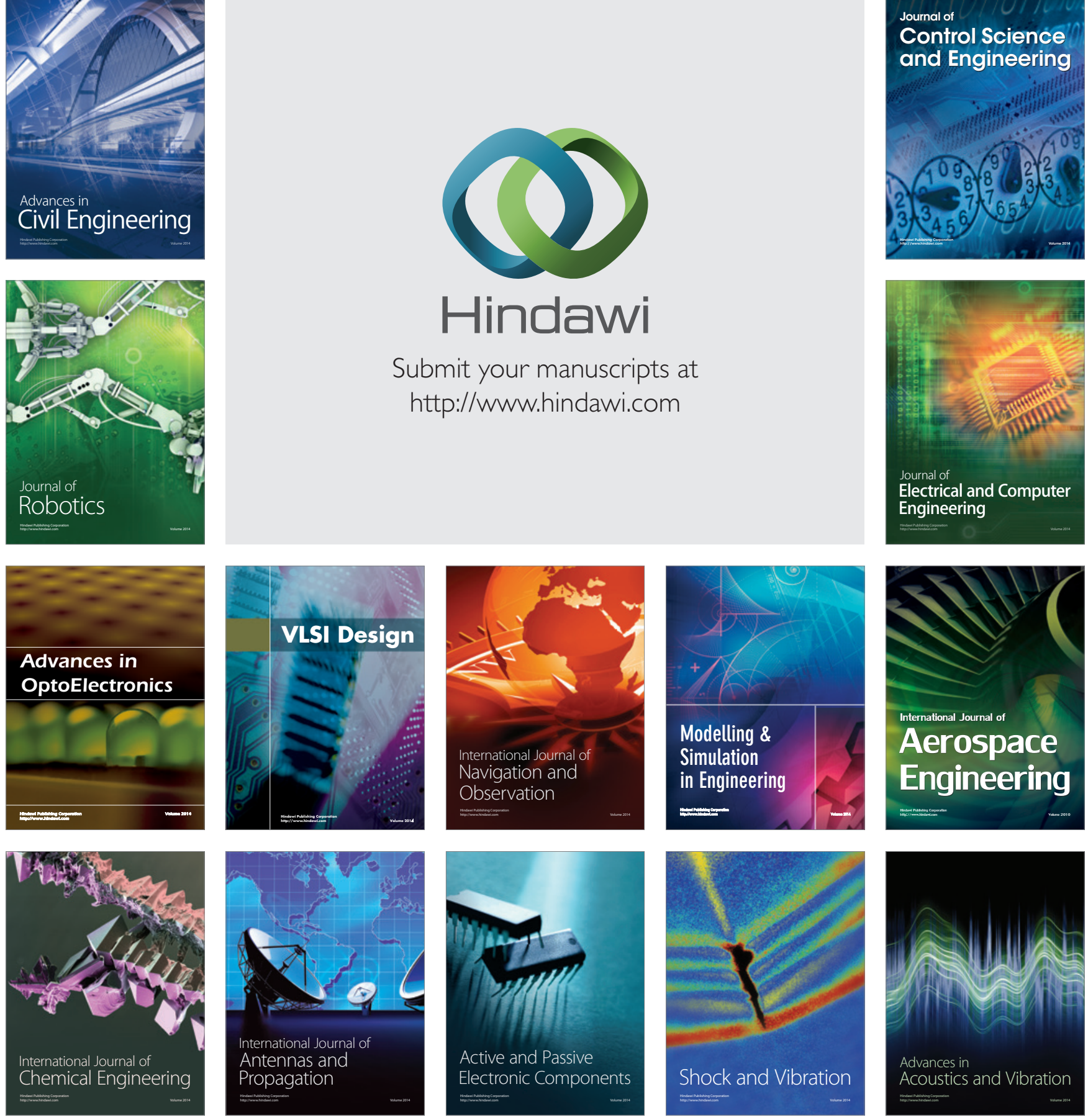